\title{
Improving CSF biomarkers' performance for predicting progression from Mild Cognitive Impairment to Alzheimer's disease by considering different confounding factors: a meta-analysis
}

\author{
Daniel Ferreira ${ }^{1}$, Amado Rivero-Santana ${ }^{2,3}$, Lilisbeth Perestelo-Pérez ${ }^{3,4,5}$ * , Eric Westman ${ }^{1}$, \\ Lars-Olof Wahlund ' ${ }^{1}$, Antonio Sarría ${ }^{3,6}$ and Pedro Serrano-Aguilar ${ }^{3,4,5}$ \\ 1 Division of Clinical Geriatrics, Department of Neurobiology, Care Sciences and Society, Karolinska Institutet, Stockholm, Sweden \\ ${ }^{2}$ Canarian Foundation of Health and Research (FUNCIS), Las Palmas de Gran Canaria, Spain \\ ${ }^{3}$ Red de Investigación en Servicios de Salud en Enfermedades Crónicas (REDISSEC), Santa Cruz de Tenerife, Spain \\ ${ }^{4}$ Evaluation Unit of the Canary Islands Health Service (SESCS), Santa Cruz de Tenerife, Spain \\ ${ }^{5}$ Center for Biomedical Research of the Canary Islands (CIBICAN), University of La Laguna, Tenerife, Spain \\ ${ }^{6}$ Agency for Health Technology Assessment (AETS), Institute of Health Carlos III, Madrid, Spain
}

\section{Edited by:}

Elena Galea, Universitat Autònoma de Barcelona, Spain

Reviewed by:

Paul Gerson Unschuld, University of Zürich, Switzerland

Esteban Hurtado, Universidad Diego

Portales, Chile

\section{${ }^{*}$ Correspondence}

Lilisbeth Perestelo-Pérez, Servicio de Evaluación del Servicio Canario de la

Salud., Camino Candelaria, s/n, El

Rosario, Santa Cruz de Tenerife

38109, Spain

e-mail:Iperperr@gobiernodecanarias. org, lilisbethp@gmail.com
Background: Cerebrospinal fluid (CSF) biomarkers' performance for predicting conversion from mild cognitive impairment (MCl) to Alzheimer's disease $(A D)$ is still suboptimal.

Objective: By considering several confounding factors we aimed to identify in which situations these CSF biomarkers can be useful.

Data Sources: A systematic review was conducted on MEDLINE, PreMedline, EMBASE, Psyclnfo, CINAHL, Cochrane, and CRD (1990-2013).

Eligibility Criteria: (1) Prospective studies of CSF biomarkers' performance for predicting conversion from $\mathrm{MCl}$ to $\mathrm{AD} /$ dementia; (2) inclusion of $\mathrm{A} \beta 42$ and T-tau and/or $p$-tau. Several meta-analyses were performed.

Results: $A \beta 42 / p$-tau ratio had high capacity to predict conversion to $A D$ in $\mathrm{MCl}$ patients younger than 70 years. The $\mathrm{p}$-tau had high capacity to identify $\mathrm{MCl}$ cases converting to $\mathrm{AD}$ in $\leq 24$ months.

Conclusions: Explaining how different confounding factors influence CSF biomarkers' predictive performance is mandatory to elaborate a definitive map of situations, where these CSF biomarkers are useful both in clinics and research.

Keywords: mild cognitive impairment, Alzheimer's disease, CSF biomarkers, confounding factors, meta-analysis, systematic review

\section{INTRODUCTION}

Mild cognitive impairment (MCI) is a high risk factor for developing dementia, particularly Alzheimer's disease (AD). About 35\% of MCI patients progress to $\mathrm{AD}$, with an annual conversion rate of 5-10\% (Mitchell, 2009). Because AD entails severe consequences, an appropriate prediction of MCI outcome is crucial for giving the patients a prognosis and to initiate therapeutical strategies as soon as possible. In this regard, the new MCI diagnostic criteria recommended by the National Institute of Aging - Alzheimer's Association (NIA-AA) emphasize the use of neuroimaging and cerebrospinal fluid (CSF) biomarkers (Albert et al., 2011). Although significant advances have been made in the field of neuroimaging, biomarkers based on CSF are at present the most convenient for studying disease progression.

The currently validated CSF biomarkers of $A D$ are $A \beta_{42}$, total $\operatorname{tau}(\mathrm{T}-\mathrm{tau})$, and phosphorylated tau (p-tau). CSF $A \beta_{42}$ is reduced, and T-tau and $\mathrm{p}$-tau levels are increased in MCI patients compared to healthy controls (Diniz et al., 2008). In addition, MCI patients with abnormal CSF biomarkers have increased risk to progress to AD (Herukka et al., 2005; Hansson et al., 2006, 2007; Bouwman et al., 2007; Brys et al., 2009; Mattsson et al., 2009; Shaw et al., 2009; Hertze et al., 2010; Buchhave et al., 2012). Buchhave et al. (2012) showed that $90 \%$ of MCI patients with pathologic CSF biomarkers developed $\mathrm{AD}$ within $9 \cdot 2$ years. This knowledge is now incorporated in the new diagnostic criteria for MCI, indicating that positive biomarkers of $A \beta$ accumulation (e.g., CSF $A \beta_{42}$ ) and neuronal injury (e.g., CSF T-tau and p-tau) confers the highest likelihood that $\mathrm{AD}$ pathophysiological processes are the cause of the cognitive dysfunction; and that individuals with this biomarker profile are more likely to decline or progress to dementia due to AD in relatively short periods (Albert et al., 2011). Regarding predictive capacity, although single CSF biomarkers have shown unsatisfactory results, their combination could be suitable to identify which MCI patients will progress to dementia 
(Ferreira et al., 2014). In particular, the $A \beta_{42} / p$-tau ratio has demonstrated high efficiency (Hansson et al., 2006; Mattsson et al., 2009; Buchhave et al., 2012; Parnetti et al., 2012; Roe et al., 2013). Two systematic reviews with meta-analysis have previously been published (Mitchell, 2009; Monge-Argilés et al., 2010). Mitchell (2009) only evaluated p-tau. Monge-Argilés et al. (2010) evaluated the three CSF biomarkers, but the group of MCI patients that converted to $\mathrm{AD}$ was compared to a mixed group of stable MCI cases and MCI patients that converted to non-AD dementias. Moreover, their analysis of combined CSF biomarkers was limited to only three studies and the combination procedure was not sufficiently detailed.

Importantly, CSF biomarkers' predictive performance could be improved by considering different confounding factors such as the MCI subtype, time to AD conversion, and age (Ferreira et al., 2014). Previous studies show that the CSF biomarkers have better predictive capacity in amnestic MCI (Vos et al., 2013), MCI patients that convert to $\mathrm{AD}$ in relatively short periods (e.g., $<12$ months) (Gaser et al., 2013), and young MCI patients (Mattsson et al., 2012). However, most of the studies performed to date do not consider these confounding factors. These aspects together with methodological variability have made it difficult to propose definitive cut-off values for CSF biomarkers. For this reason, the fact of disseminating the use of CSF biomarkers to clinical routine is compromised at present (Ferreira et al., 2014).

The main objective in this study was to carefully evaluate the capacity of the CSF biomarkers to predict conversion from MCI to $\mathrm{AD}$ in several clinically relevant situations. In particular, we aimed to identify for which specific MCI patients these CSF biomarkers might be useful in clinical practice. In order to address this question, several meta-analyses were performed for studies that prospectively analyzed the predictive performance of CSF $A \beta_{42}$ and T-tau and/or p-tau. The design of the included studies is baseline cross-sectional comparisons between MCI patients that convert to AD or dementia (MCI-C) and MCI patients that remain stable (MCI-S) at follow-up. We hypothesized that combined CSF biomarkers would have better predictive performance than single CSF biomarkers, and that this performance could be increased by controlling for different confounding factors such as the MCI subtype, time to AD conversion, and age, among others.

\section{MATERIAL AND METHODS SEARCH STRATEGY AND SELECTION CRITERIA}

A systematic review was conducted for the period between January 1990 and September 2013 in the following electronic databases: MEDLINE and PreMedline, EMBASE, PsycInfo, CINAHL, Cochrane Library, and CRD. The search strategy was developed for each database using the following Medical Subject Heading (MeSH) and free-text terms: "Alzheimer's disease diagnosis" or "Alzheimer's disease," and "abeta-42" or "T-tau” or "P-tau" or "tau" or "phospho-tau" or "phosphorylated tau." Examples for the two major databases are shown in Table S1 in Supplementary Materials (MEDLINE) and Table S2 in Supplementary Materials (EMBASE). In addition, reference sections were searched to identify relevant publications.

Inclusion criteria for this meta-analysis were studies that (1) performed a prospective analysis of the CSF biomarkers'

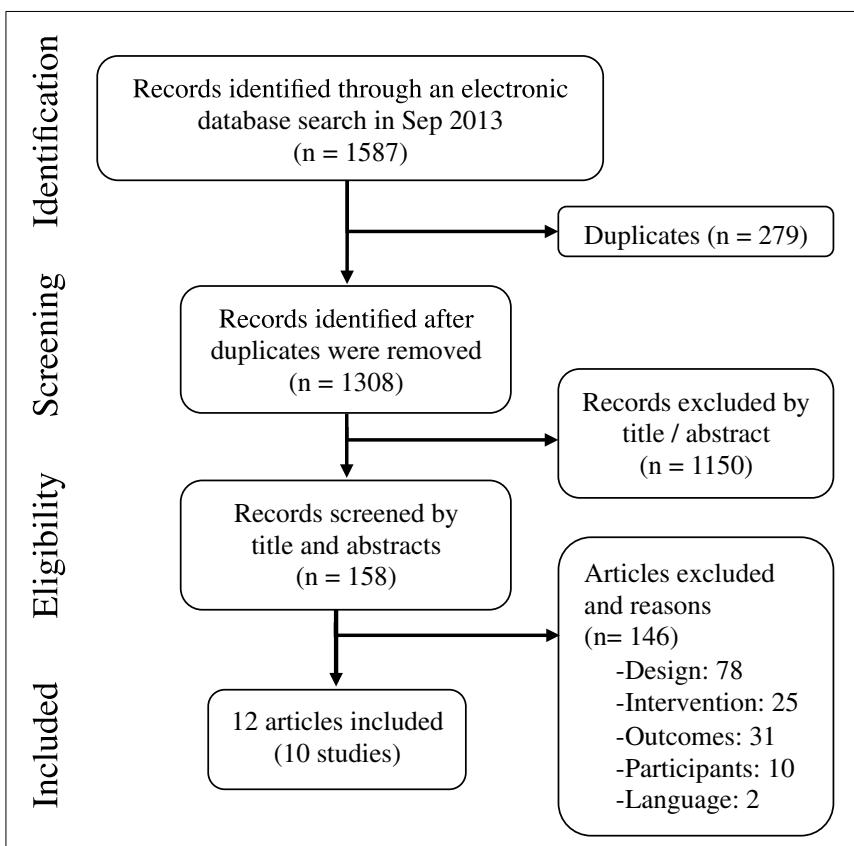

FIGURE 1 | Study selection flow

performance for predicting conversion to $\mathrm{AD}$ or dementia in individuals with MCI at baseline; (2) included at least two CSF biomarkers, being $\mathrm{A} \beta_{42}$ always required along with T-tau and/or p-tau; and (3) were published in English or Spanish. Studies were excluded if they did not report sensitivity or specificity values, or any other data that enabled its calculation. Two reviewers independently performed the study selection (Daniel Ferreira and Amado Rivero-Santana), and in case of doubt and/or disagreements a third reviewer was consulted (Lilisbeth Perestelo-Pérez). The search yielded 1308 references after discarding duplicates. One-hundred fifty-eight articles were selected by title and abstract. After applying eligibility criteria, 12 articles were eventually included (Hampel et al., 2004; Herukka et al., 2005; Parnetti et al., 2006, 2012; Eckerström et al., 2010; Hertze et al., 2010; Monge-Argilés et al., 2011; Buchhave et al., 2012; Ewers et al., 2012; Gaser et al., 2013; Toledo et al., 2013; Vos et al., 2013). Three of these studies included data from the Alzheimer's Disease Neuroimaging Initiative (ADNI). As these studies represent different analyses of overlapping ADNI subsamples, only one ADNI study was included for each metaanalysis depending on the analyzed biomarker. If two ADNI studies were available for the same biomarker, the one with largest sample was selected. Selection flow including reasons for study exclusion at each phase is shown in Figure 1.

\section{DATA COLLECTION, RISK OF BIAS, AND EVALUATION OF METHODOLOGICAL OUALITY}

A data extraction sheet was developed to collect relevant data by covering: author and publication year, country, objectives, methods (with special attention to participants' recruitment procedures, study design, follow-up length, CSF biomarkers evaluated including $A \beta_{42} /$ T-tau and $A \beta_{42} / p$-tau ratios, diagnostic groups characteristics, and statistical analyses), results, and conclusions. 
Data extraction was carried out by two researchers (Daniel Ferreira and Amado Rivero-Santana), and quality and accuracy of the extraction was verified by a third researcher (Lilisbeth Perestelo-Pérez).

Several strategies were followed in order to reduce the risk of bias related to publication, data availability, and reviewer selection (see Table S3 in Supplementary Materials). The QUADAS2 scale (Whiting et al., 2011) was used in order to assess the methodological quality of the included studies. The scale was applied by two researchers (Amado Rivero-Santana and Daniel Ferreira), and in case of doubt and/or disagreements a third was consulted (Lilisbeth Perestelo-Pérez). Finally, this study was performed in accordance with the PRISMA statement (Liberati et al., 2009; Moher et al., 2010), which provides a detailed guideline of preferred reporting style for systematic reviews and meta-analyses.

\section{IDENTIFICATION OF POTENTIAL CONFOUNDING FACTORS OF CSF BIOMARKERS' PREDICTIVE PERFORMANCE}

We hypothesized that CSF biomarkers' predictive performance might be influenced by different confounding factors. To explore sources of heterogeneity, the following factors were defined a priori based on the literature (see Table 1 for a detailed description of the different factors and considered categories): (1) recruitment setting; (2) MCI subtype; (3) diagnostic criteria for MCI at baseline; (4) diagnostic criteria for AD at follow-up; (5) postmortem confirmation of AD pathology; (6) criteria for conversion from MCI to $\mathrm{AD} /$ dementia; (7) diagnosis at follow-up; (8) follow-up length (as rough estimation of time to AD conversion); (9-11) MCI severity at baseline according to mini-mental state examination (MMSE), clinical rating [e.g., clinical dementia rating (CDR), global deterioration scale (GDS)], and magnetic resonance imaging (MRI) rating (i.e., degree of brain atrophy); (12) Age; (13) gender distribution; (14) years of education; (15) family history of AD; (16) APOE e4 status; (17) technology applied for the CSF analysis; and (18) cut-offs for interpreting the CSF levels.

\section{STATISTICAL ANALYSIS}

For each article, true and false positives/negatives values were calculated from sensitivity, specificity, positive predicted value, negative predicted value, and/or the rate of converters and nonconverters. A global meta-analysis was performed for each single CSF biomarker (i.e., $\mathrm{A} \beta_{42}$, T-tau, and p-tau) and two relevant ratios (i.e., $A \beta_{42} / \mathrm{T}$-tau and $\mathrm{A} \beta_{42} / \mathrm{p}$-tau). Analyses were performed with the MetaDisc 1.1.1 software (Zamora et al., 2006). Sensitivity and specificity pooled estimates were calculated with random-effects models (DerSimonian and Laird, 1986), which yield more conservative estimates. For a qualitative interpretation of sensitivity and specificity results, values above $80 \%$ were considered indicative of satisfactory predictive performance according to international recommendations (The Ronald and Nancy Reagan Research Institute of the Alzheimer's Association and the national Institute on Aging working Group, 1998). Positive and negative likelihood ratios were calculated from resulting sensitivity and specificity values and interpreted following established guidelines (see these guidelines in Figure 2 footnotes) (Qizilbash, 2002). Likelihood ratios indicate how the pretest probability of disease is increased or decreased by the outcome of a diagnostic test. A positive likelihood ratio $[\mathrm{LR}+=\operatorname{sensitivity} /(1-$ specificity $)]$ greater than one increases the probability that the disease is present (in this context progression to $\mathrm{AD}$ ) and helps to rule-in MCI-C cases. A negative likelihood ratio (LR- $=(1-$ sensitivity $) /$ specificity) of less than one diminishes the probability that disease is present and helps to rule-out MCI-C cases. Statistical heterogeneity was explored with the Cochran Q-test. As this statistic has low power when few studies are available, a recommended $p$-value of $0 \cdot 10$ was established as statistical significance threshold to detect heterogeneity (Hardy and Thompson, 1998). Differences in sensitivity and specificity values for pairs of subgroup meta-analyses (e.g., MCI cases younger than 70 years vs. older than 70 years) were tested with the formula: $Q_{\mathrm{BET}}=Q_{\mathrm{TOT}}-\left(Q_{1}+Q_{2}\right)$. Where $Q_{\mathrm{TOT}}$ represents the overall inter-study variability, and $Q_{1}$ and $Q_{2}$ represents inter-study variability for each subgroup in the comparison

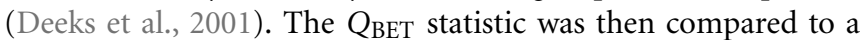
$\chi^{2}$ distribution with $J-1$ degrees of freedom using a significance level of $0 \cdot 05$, where $J$ is the number of subgroups.

\section{RESULTS \\ MAIN CHARACTERISTICS OF INCLUDED STUDIES AND METHODOLOGICAL QUALITY}

Among the 12 studies included, 10 offered data about the diagnostic performance of $A \beta_{42}, 6$ about T-tau, 5 about p-tau, and 6 about the $A \beta_{42} /$ T-tau and $A \beta_{42} / p$-tau ratios. Main study characteristics are detailed in Table 2. Methodological quality (QUADAS-2) is shown in Table S4 in Supplementary Materials. In summary, (1) Patient selection: only two studies demonstrated low risk of bias; seven did not explicitly state consecutive or random samples; patients could have been inappropriately excluded in nine studies. (2) Diagnostic test: seven studies proved low risk of bias by using external cut-off values or establishing the cut-off in the study sample independently of the clinical diagnosis [i.e., mixture model analysis in Buchhave et al., 2012]. (3) Diagnostic criterion: all the studies were classified as unclear given that postmortem confirmation of AD pathology was never performed. (4) Patients flow and follow-up: three studies demonstrated low risk of bias; all the studies applied the same reference standard to all the patients, but patients were followed during only two years or less in five studies; six studies did not include all baseline patients in the final analyses.

\section{GLOBAL META-ANALYSIS: CSF BIOMARKERS' PREDICTIVE PERFORMANCE}

Table 3 shows sensitivity and specificity values with 95\% CI, heterogeneity, and likelihood ratios. Heterogeneity was significant for the three single biomarkers as well as for the two evaluated ratios. $\mathrm{A} \beta_{42} / \mathrm{p}$-tau ratio showed the best performance with $85 \%$ sensitivity, $79 \%$ specificity, and a negative likelihood ratio of $0 \cdot 19$, indicating moderate decrease in the probability that the disease is present.

\section{SUBGROUPS META-ANALYSES: IDENTIFICATION OF CLINICAL SITUATIONS WITH INCREASED CSF BIOMARKERS' PREDICTIVE PERFORMANCE}

Table 1 shows the list of potential confounding factors defined a priori based on the literature. Due to the low variability across the 
Table 1 | Potential confounding factors of CSF biomarkers' predictive performance.

\begin{tabular}{|c|c|}
\hline Factors & Considered categories \\
\hline Setting & Specialized centers $(9+1)$ vs. primary care $(0)$ vs. general population (0) \\
\hline $\mathrm{MCl}$ subtype & Amnestic $\mathrm{MCl}(6+1)$ vs. non-amnestic $\mathrm{MCl}(1)$ vs. mixed sample $(2)^{\mathrm{a}}$ vs. non-specified (1) \\
\hline Diagnostic criteria for $\mathrm{MCl}$ at baseline & $\begin{array}{l}\text { Petersen et al. (1999, 2001); Petersen, } 2004 \text { or Petersen's group } 2006^{b} \text { (8) vs. Winblad et al. (2004) (1) vs. } \\
\text { ADNI criteria (+1) }\end{array}$ \\
\hline Diagnostic criteria for $\mathrm{AD}$ at follow-up & NINCDS-ADRDA $(8+1)$ vs. non-specified (1) \\
\hline Postmortem confirmation of AD pathology & All the studies lacked postmortem confirmation of AD pathology (0) \\
\hline Diagnosis at follow-up & $A D(6+1)$ vs. mixed group of dementias $(3)^{c}$ \\
\hline Criteria for conversion & Fulfillment of diagnostic criteria (7+ 1) vs. Decline in clinical scales (e.g., CDR) (1) vs. non-specified (1) \\
\hline
\end{tabular}

MCI SEVERITY AT BASELINE

MMSE total score

No enough variability: all studies reporting MMSE have mean scores between 23 and $30(7+1)$ vs. non-specified (2)

Clinical rating

$\mathrm{CDR}=0.5 / \mathrm{GDS}=3(5+1)$ vs. Non-specified (4)

$\mathrm{MRI}$ rating

Only available for $2(+1)$ studies, with variability in procedures

\section{DEMOGRAPHICS/RISK FACTORS AT BASELINE}

Age

Gender

Education

Family history of $A D$

APOE e4 status $\leq 70$ years (4) vs. $>70$ years $(4+1)$ vs. non-specified (1)

Results are never presented separately for men and women (0)

$\leq 12$ years (3) vs. $>12$ years (+1) vs. non-specified (6)

Not enough information (1)

Not enough information $(3+1)$

\section{CSF METHODS}

Technology for CSF analysis

Cut-offs for interpreting CSF levels
ELISA (6) vs. XMAP $(3+1)$

Great variability: Internal (highest value of SN + SP or Youden's Index) (3) vs. External or independent of clinical diagnosis (Mixture model analysis, obtained from another cohort in the same study; Hulstaert et al., 1999; Sjögren et al., 2001; Shaw et al., 2009; Zetterberg et al., 2003) (4 + 1) vs. both internal and external (2)

Between brackets, number of studies available for at least one biomarker; +1 refers to ADNI studies (only one ADNI study is included for each subgroup meta-analysis). ${ }^{a}$ Herukka et al. (2005) included amnestic MCl patients as well as patients with other types of cognitive decline. Monge-Argilés et al. (2011) included a mixed group of amnestic $\mathrm{MCl}$ and non-amnestic $\mathrm{MCl}$ patients.

${ }^{b}$ Diagnostic criteria for MCl published by Petersen's group in Artero et al. (2006).

${ }^{c}$ Both in Parnetti et al. (2006) and Monge-Argilés et al. (2011) it is unclear whether MCl converters progressed exclusively to AD.

$M C l$, mild cognitive impairment; $A D$, Alzheimer's disease; MMSE, mini-mental state examination; MRI, magnetic resonance imaging; APOE, apolipoprotein E; CSF, cerebrospinal fluid; ADNI: Alzheimer's disease neuroimaging initiative; NINCDS-ADRDA, National Institute of Neurological and Communicative Disorders and Stroke and the Alzheimer's Disease and Related Disorders Association; CDR, clinical dementia rating; GDS, global deterioration scale.

studies, it was only possible to perform subgroups meta-analyses for the following factors: (1) MCI subtype (studies exclusively including amnestic MCI cases); (2) diagnosis at follow-up (studies including MCI patients that converted exclusively to AD); (3) follow-up length (as rough estimation of time to $\mathrm{AD}$ conversion: $\leq 24$ months vs. $>24$ months); (4) age (studies with mean age younger vs. older than 70 years); and (5) technology for CSF analysis (ELISA vs. xMAP). As controlled factors, all the studies included samples from specialized centers; lacked postmortem $\mathrm{AD}$ confirmation; used comparable diagnostic criteria for MCI; applied NINCDS-ADRDA criteria for AD diagnosis [except one study (Herukka et al., 2005)]; and included MCI cases with similar global cognitive status (i.e., MMSE). Information on MCI severity according to MRI rating, gender distribution, level of education, family history of $\mathrm{AD}$, and $\mathrm{APOE}$ e4 status was scarce or absent. 


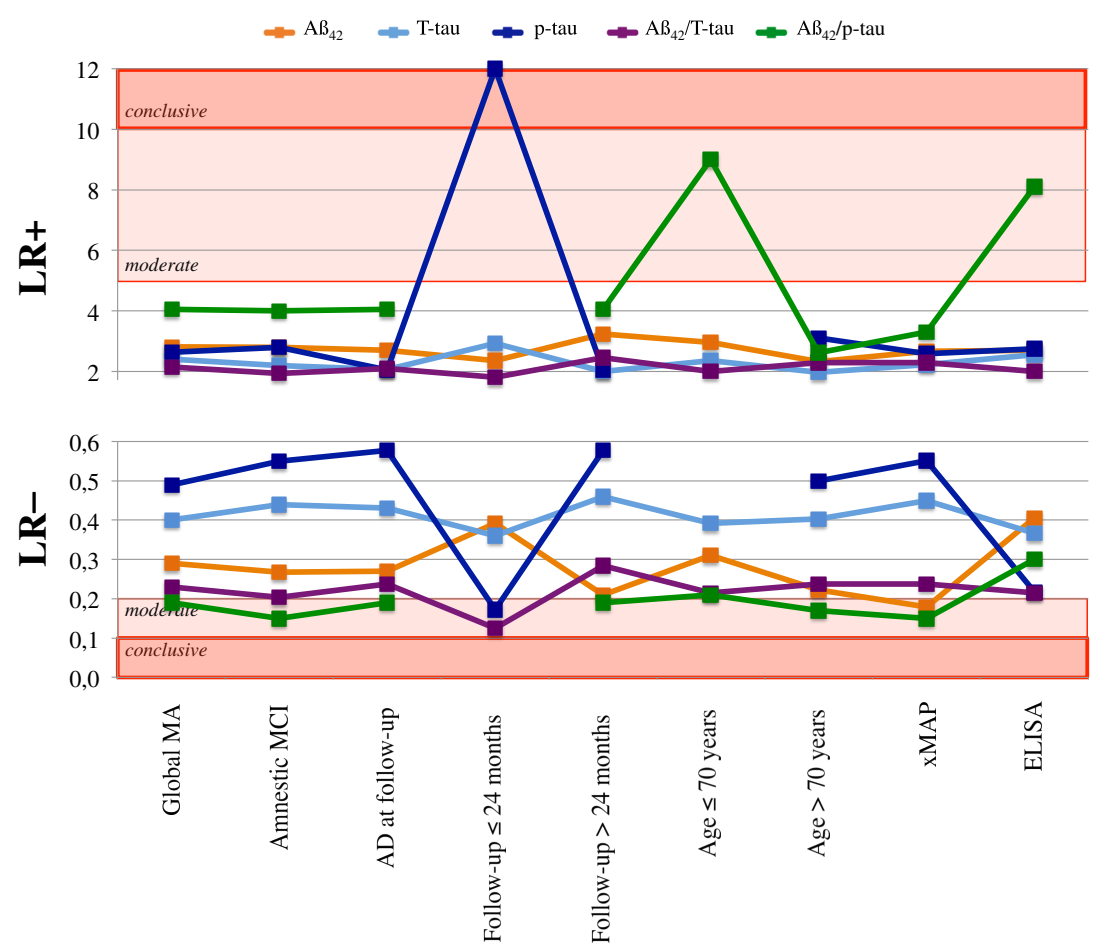

FIGURE 2 | Positive and negative likelihood ratios. A LR+ greater than one increases the pretest probability that the disease is present [in this context progression from $\mathrm{MCl}$ to $\mathrm{AD}$ or, in other words, $\mathrm{MCl}$ due to $\mathrm{AD}$ (Albert et al., 2011)]. A LR- of less than one diminishes the pretest probability that disease is present. The established guidelines (Qizilbash, 2002) states that a LR+ greater than 10 will often make conclusive changes to the pretest probability, indicating that the disease is likely present; a LR+ between 5 and 10 corresponds to moderate increase in probability; and a LR+ between 2 and 5 corresponds to small increase. A LR - of less than 0.1 will often make conclusive changes to the pretest probability that the disease is present, indicating that the disease is unlikely present; a LR- between 0.1 and 0.2 corresponds to moderate decrease in probability; and a LR - between 0.2 and 0.5 corresponds to small decrease. LR+, positive likelihood ratio; LR-, negative likelihood ratio; Global MA, global meta-analysis; $\mathrm{MCl}$, mild cognitive impairment; $A D$, Alzheimer's disease.
Table 3 shows sensitivity and specificity values with 95\% $\mathrm{CI}$, inter-groups difference $\left(\mathrm{Q}_{\mathrm{BET}}\right)$, heterogeneity, and likelihood ratios. Heterogeneity was significant in most of the subgroups meta-analyses. Noteworthy, CSF biomarkers' predictive performance was optimal $(>80 \%)$ in two clinically relevant situations, and heterogeneity was no longer significant: (1) P-tau alone had $84 \%$ sensitivity and $93 \%$ specificity for MCI cases converting to $\mathrm{AD}$ in $\leq 24$ months, significantly different from $59 \%$ sensitivity $(p=0.01)$ and $71 \%$ specificity $(p<0.001)$ in studies with followup periods $>24$ months; (2) $\mathrm{A} \beta_{42} / \mathrm{p}$-tau ratio showed $81 \%$ sensitivity and $91 \%$ specificity in MCI patients younger than 70 years, significantly different from $66 \%$ specificity in MCI patients older than 70 years $(p<0.001)$.

$\mathrm{A} \beta_{42} / \mathrm{p}$-tau ratio showed the best performance across the different subgroups meta-analyses. Sensitivity was slightly increased in studies including only amnestic MCI cases (heterogeneity no longer significant), MCI patients older than 70 years (heterogeneity no longer significant), and studies using ELISA. A $\beta_{42} / \mathrm{T}$-tau ratio yielded optimal sensitivity values, but suboptimal specificity. Results were not satisfactory for single CSF biomarkers, except for the remarkably good p-tau diagnostic performance commented above.
The analysis of positive likelihood ratios showed extremely high increase in the probability that the disease is present $(\mathrm{LR}+=12)$ for $\mathrm{p}$-tau in $\mathrm{MCI}$ cases converting to $\mathrm{AD}$ in $\leq 24$ months (Figure 2; Table 3). Moreover, there was a moderate increase in the probability that the disease is present $(\mathrm{LR}+=5-10)$ for the $\mathrm{A} \beta_{42} / \mathrm{p}$-tau ratio in two situations: $\mathrm{MCI}$ patients younger than 70 years; and studies using ELISA technology. The analysis of negative likelihood ratios showed moderate decrease in the probability that the disease is present $(\mathrm{LR}-=0.1-0.2)$ in several situations: $\mathrm{p}$-tau and $\mathrm{A} \beta_{42}$ /T-tau ratio in MCI cases converting to $\mathrm{AD}$ in $\leq 24$ months; $\mathrm{A} \beta_{42} / \mathrm{p}$-tau ratio in the global meta-analysis as well as in all the subgroups meta-analyses (except in MCI patients younger than 70 years and studies using ELISA technology).

\section{DISCUSSION}

The two main findings in this study are that the $A \beta_{42} / p$-tau ratio has high capacity to predict $\mathrm{AD}$ conversion in MCI patients younger than 70 years; and p-tau alone has high capacity to identify MCI cases converting to AD in $\leq 24$ months. The analysis of likelihood ratios showed that, in both situations, a CSF test result indicating pathological values of $A \beta_{42} / \mathrm{p}$-tau or p-tau significantly increase the probability that the disease is present [in this context 


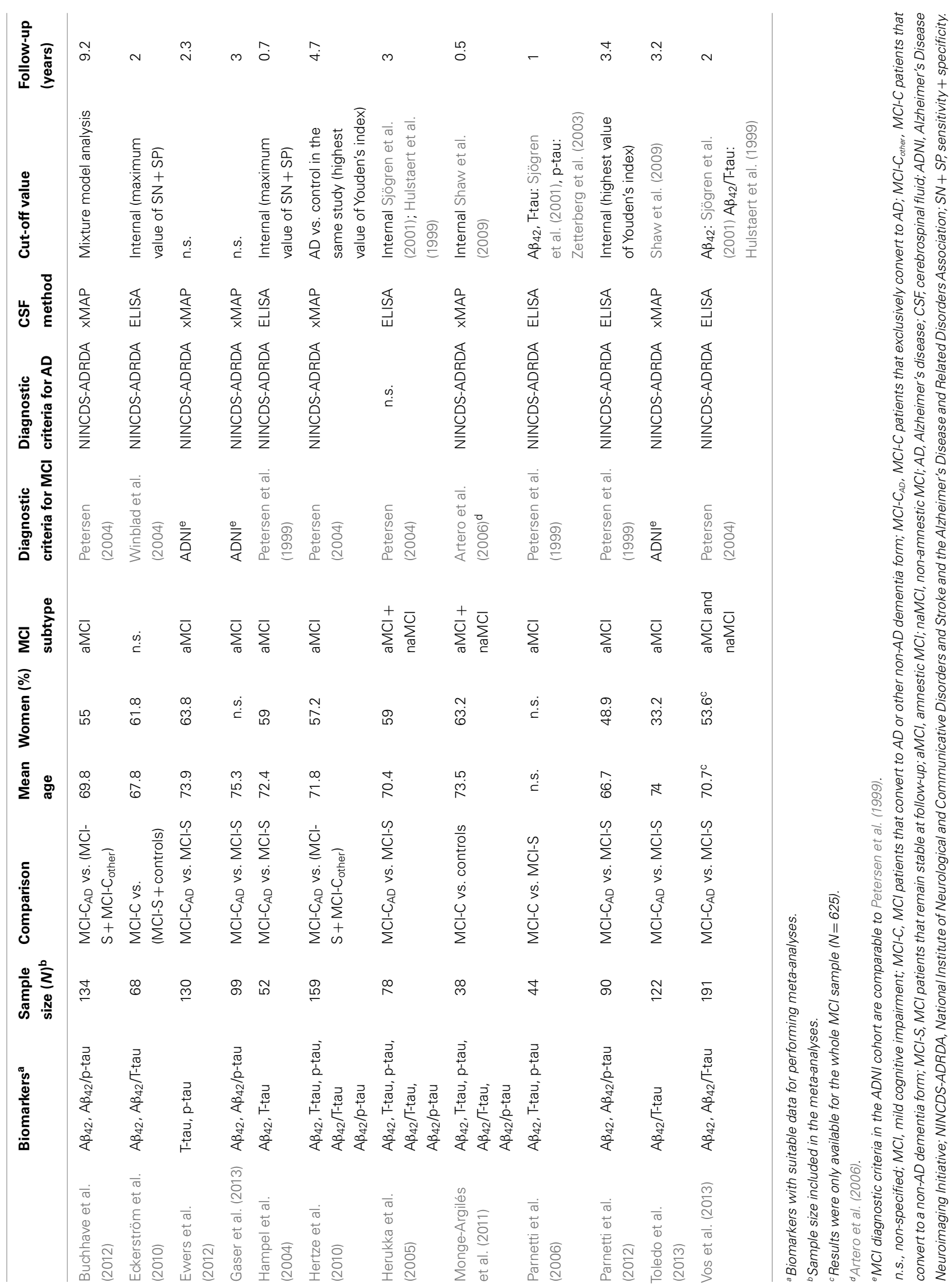


Table 3 | Global meta-analysis and subgroups meta-analyses.

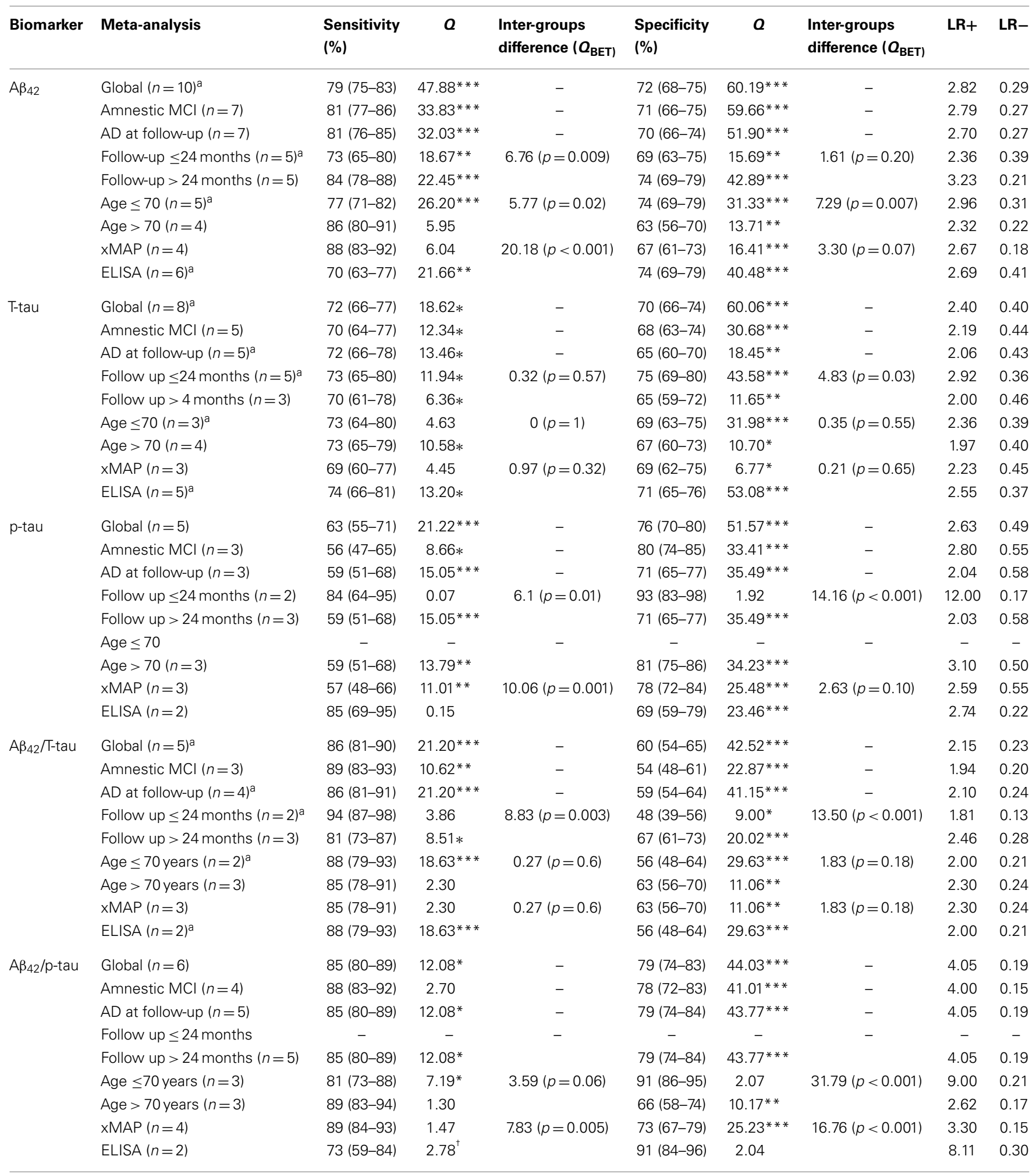

${ }^{+} p<0.10 ;{ }^{*} p<0.05 ;{ }^{*} p<0.01 ;{ }^{* *} p<0.001$.

${ }^{a}$ Number of estimations is $n+1$ due to results were reported separately for amnestic $\mathrm{MCl}$ and non-amnestic $\mathrm{MCl}$ patients in Vos et al. (2013).

$Q$, heterogeneity (Cochran Q-test); LR+, positive likelihood ratio; $L R-$, negative likelihood ratio; Global, global meta-analysis; $n$, number of studies included in the meta-analysis; $M C l$, mild cognitive impairment; $A D$, Alzheimer's disease. 
progression from $\mathrm{MCI}$ to $\mathrm{AD}$ or, in other words, $\mathrm{MCI}$ due to $\mathrm{AD}$ (Albert et al., 2011)].

Better predictive performance of the CSF biomarkers in younger MCI patients has been recently shown in a large multicenter study (Mattsson et al., 2012). A fact that may explain this result is that typical $\mathrm{AD}$ brain alterations increase with age in individuals without dementia (Green et al., 2000; Bennett et al., 2006), with about a third of cognitively normal elderly evidencing an AD-like pattern of CSF biomarker alterations (Ewers et al., 2007; Bouwman et al., 2009; Mattsson et al., 2009; Shaw et al., 2009). This also occurs in stable MCI cases, therefore obstructing specificity for $\mathrm{AD}$ and undermining CSF biomarkers' performance.

Regarding time to AD conversion, Gaser et al. (2013) showed that the CSF biomarkers had generally better performance for MCI cases that converted to $\mathrm{AD}$ in $<12$ months as compared with $\mathrm{MCI}$ cases that converted to $\mathrm{AD}$ in $>12$ months. In the current metaanalysis, this finding is still valid when considering 24 months as threshold. However, Buchhave et al. (2012) reported that the combination of CSF biomarkers might not be recommendable at 60 months before $\mathrm{AD}$ conversion. The reason for this is that at that point, many MCI-C have normal T-tau levels but already pathological $\mathrm{A} \beta_{42}$ levels. In another study, the combination of CSF biomarkers with structural MRI showed $>80 \%$ sensitivity during the first 18 months of follow-up, decreasing to $75 \%$ at 24 months, and to $68 \%$ at 36 months (Westman et al., 2012). Therefore, predictive value and biomarkers' utility strongly depend on the stage of the disease and time to conversion. $A \beta_{42}$ performs better than Tau 5-10 years before conversion to $\mathrm{AD}$, but T-tau and $\mathrm{p}$-tau have better predictive power $0-5$ years before conversion (Buchhave et al., 2012). Other biomarkers such as those based on structural MRI have the highest performance the closer to AD diagnosis. Future research should thus pursue in combining the CSF biomarkers not only with each other but also with other biomarkers. Recent studies show an increase in the diagnostic efficiency of CSF biomarkers when combined with neuroimaging biomarkers (Vos et al., 2012; Westman et al., 2012; Choo et al., 2013; Galluzzi et al., 2013; Prestia et al., 2013; Shaffer et al., 2013). The development of new combinations and indexes may contribute not only to predict $\mathrm{AD}$ conversion but, importantly, to facilitate prediction of time to conversion, which is still challenging.

Importantly, the $A \beta_{42} / p$-tau ratio showed satisfactory predictive performance in a heterogeneous group of MCI patients, which better represents the clinical reality (global meta-analysis). Moreover, it is noteworthy that the sensitivity was increased in two specific conditions: amnestic MCI patients and old MCI patients (>70 years). Recently, Vos et al. (2013) showed that the CSF biomarkers are more sensitive in amnestic MCI than in non-amnestic MCI patients. An explanation for this is MCI heterogeneity. Only $30-60 \%$ of the MCI patients are affected by prodromal AD, whereas the others may stem from a variety of different etiologies and pathologies (Ritchie et al., 2001; Petersen, 2004). The amnestic subtype is mainly associated with $\mathrm{AD}$ pathology. Nonetheless, vascular etiology has also been referred as explicative factor, especially in those cases with cognitive impairment encompassing other domains besides memory (Petersen, 2004; Winblad et al., 2004). On the contrary, the non-amnestic subtype may have higher likelihood of progressing to non-AD dementias such as dementia with Lewy bodies or frontotemporal lobar degeneration (Petersen, 2004; Winblad et al., 2004). In this regard, it seems reasonable that the CSF biomarkers validated for AD perform better in the amnestic MCI cases. In agreement with Vos et al. (2013), this may have implications for clinical implementation of the new revised criteria for MCI (Albert et al., 2011), given that both amnestic and non-amnestic subtypes are considered in this criteria as possible prodromal stages of AD-type dementia. Regarding the finding of better CSF biomarkers' sensitivity in old MCI patients, this is in line with the discussion above about the age-related increase in AD-like CSF biomarker patterns. Mattsson et al., 2012 also found increased sensitivity in MCI patients older tan 65 years compared to MCI patients younger than 65 years. On the other hand, $\mathrm{A} \beta_{42} / \mathrm{p}$-tau specificity was not increased in any of the subgroups meta-analyses except for young MCI patients ( $\leq 70$ years), as discussed above. The three CSF biomarkers alone and the $\mathrm{A} \beta_{42} / \mathrm{T}$-tau ratio showed suboptimal predictive power except $\mathrm{p}$-tau for $\mathrm{MCI}$ cases converting to $\mathrm{AD}$ in $\leq 24$ months, as already commented.

A better performance of the $A \beta_{42} / p$-tau ratio over the other CSF biomarkers has been reported in previous studies on MCI prediction (Hansson et al., 2006; Mattsson et al., 2009; Buchhave et al., 2012; Parnetti et al., 2012; Roe et al., 2013) and differential diagnosis between $\mathrm{AD}$ and other dementias (Maddalena et al., 2003; Jong et al., 2006; Holtzman, 2011). This finding is likely due to this ratio reflects two aspects of $A D$ pathology, i.e., plaques $\left(A \beta_{42}\right)$, and neurodegeneration (tau). Moreover, $\mathrm{p}$-tau usually shows better performance than T-tau (Mitchell, 2009; Bloudek et al., 2011; van Harten et al., 2011), probably because p-tau is not only a marker of axonal damage and neuronal degeneration, as T-tau, but it is more closely related to $\mathrm{AD}$ pathophysiology and the formation of neurofibrillary tangles (Anoop et al., 2010; Holtzman, 2011). In addition, CSF p-tau concentrations in dementia with Lewy bodies, frontotemporal lobar degeneration, and vascular dementia have been referred to be more comparable to concentrations in controls than to concentrations in AD patients (van Harten et al., 2011). This positively affects prediction of MCI due to AD.

Regarding the clinical value of the CSF biomarkers, results for negative likelihood ratios were normally better than results for positive likelihood ratios. This means that the CSF biomarkers are more useful to identify MCI patients that remain stable at followup (MCI-S) than to rule-in MCI patients that will progress to $\mathrm{AD}$ or dementia (MCI-C). This finding supports the consideration made in the new MCI diagnostic criteria in relation to biomarkers profile suggesting that the MCI syndrome is unlikely to be due to $\mathrm{AD}$ (point 3.6.4. in Albert et al. (2011): "the definitive absence of evidence of either $A \beta$ deposition or neuronal injury strongly suggests that the MCI syndrome is not due to AD"). Our study shows that a normal result in the $\mathrm{A} \beta_{42} / \mathrm{p}$-tau ratio has a moderate decrease in the probability that the disease is present (conversion to $\mathrm{AD}$ ). This is true in all the situations evaluated in the different subgroups meta-analyses, although we could not confirm this for MCI cases converting to AD in $\leq 24$ months because only one study was available (Monge-Argilés et al., 2011). This single study reported $86 \%$ sensitivity and $75 \%$ specificity (Monge-Argilés et al., 2011). Therefore, it is quite probable that a meta-analysis of the $\mathrm{A} \beta_{42} / \mathrm{p}$-tau ratio in $\mathrm{MCI}$ cases converting to $\mathrm{AD}$ in $\leq 24$ months would provide a satisfactory negative likelihood ratio, given that 
both p-tau and the $A \beta_{42} /$ T-tau ratio showed optimal results. On the other hand, positive likelihood ratios were normally within the range of a small increase in the probability that the disease is present. The only two situations where conclusive increase was achieved are those already commented above $\left(\mathrm{A} \beta_{42} / \mathrm{p}\right.$-tau ratio in young MCI patients and p-tau in MCI cases converting to $\mathrm{AD}$ in $\leq 24$ months). This finding may have implications for the consideration made in the new MCI diagnostic criteria regarding biomarkers pattern indicating a high likelihood that the MCI syndrome is due to $\mathrm{AD}$ [point 3.6.1. in Albert et al., 2011]. In particular, young MCI patients with positive biomarkers of $A \beta$ accumulation and neuronal injury seems to have increased risk to decline or progress to dementia due to AD in relatively short periods.

To determine in which specific situations the CSF biomarkers provide satisfactory predictive performance is of great relevance. In this study, some of those situations have been identified. However, despite these positive results, we acknowledge that much additional work needs to be done to validate the application of the CSF biomarkers as they are proposed in the new revised criteria for MCI (Albert et al., 2011). The main limitation for extending the use of the CSF biomarkers to the clinical routine is the difficulty to establish appropriate cut-offs. There is a big variability in the cut-offs applied across the different studies. This is in part related to differences in methodological aspects as well as absence of technical standardization. In this meta-analysis, two aspects related to variability in CSF methods were considered. First, we tried to analyze the influence of different cut-offs for the CSF biomarkers. Due to the great variability found it was not possible to group the studies in order to perform specific subgroups meta-analyses (Table 1). Second, the technology for the CSF analysis applied was also considered as potential confounding factor. Results showed that sensitivity and specificity values differed depending on whether xMAP (Luminex, Austin, US) or ELISA (Innogenetics, Ghent, Belgium) technology was used. A clear pattern was not found however. Therefore, future research is mandatory to hopefully ascertain universal cut-offs values for the CSF biomarkers. Several studies indicate that the standardization of laboratory procedures could contribute to reduce variability in the results (Hansson et al., 2006; Fagan et al., 2011; Mattsson et al., 2011).

Therefore, standardization of methodological aspects is expected to increase the clinical utility of the CSF biomarkers. In this meta-analysis, we demonstrate that several confounding factors are another source of variability in published diagnostic/predictive performance and cut-offs. We show that CSF biomarkers' performance can be improved and heterogeneity reduced by carefully considering these confounding factors. In this regard, future studies should be addressed to explain how these factors influence the diagnostic and predictive performance of the CSF biomarkers. This need is reinforced by the fact that we could not evaluate 13 of the 18 identified potentially confounding factors given the lack of studies directly addressing these aspects. A related limitation is the scarce number of studies available for some of the analyses. This causes that certain subgroups meta-analyses could be influenced by some of the other confounding factors. In order to evaluate this, an analysis of coincident studies across factors was performed. Table S5 in Supplementary Material shows that most of the subgroups were rather independent from each other. However, for $\mathrm{p}$-tau and $\mathrm{A} \beta_{42} / \mathrm{p}$-tau, studies including follow-up periods $>24$ months coincided with studies with $\mathrm{AD}$ diagnosis at follow-up; and for $\mathrm{p}$-tau and $\mathrm{A} \beta_{42} / \mathrm{T}$-tau, studies using xMAP technology coincided with studies including MCI cases older than 70 years (and vice versa only for $\mathrm{A} \beta_{42}$ /T-tau: ELISA technology with studies including MCI cases younger than 70 years). Another limitation is that systematic reviews and meta-analyses are essential tools for summarizing evidence accurately and reliably, but might be susceptible of bias if not properly conducted. Following PRISMA recommendations (Liberati et al., 2009; Moher et al., 2010), several strategies were carefully considered in this study to reduce risk of bias related to publication, data availability, and reviewer selection. Evidence was rigorously reviewed and literature was supplemented with manual query of relevant studies in order to minimize both publication and reviewer selection bias. Selected studies were carefully examined for clues suggesting that there may be missing results or data. Moreover, assessments were completed independently by more than one reviewer and consensus was required. Regarding the included studies, QUADAS-2 was applied to evaluate risk of bias and results applicability. It must be noticed that "domain 1" indicated high probability of patient selection bias in six of the included studies, related to inclusion of not completely consecutive or random samples, and not perfect avoidance of inappropriate exclusions. In particular, patients were normally selected from specialized centers on the basis of availability of CSF data, a procedure not generally performed in all incoming patients. This fact, may have certain impact in the applicability of the results, although these six studies scored rather well in the other three domains, indicating that the index test, the standard test, and flow and timing are not compromised. Another drawback is that the follow-up period was used as rough measure of time to $\mathrm{AD}$ conversion. Therefore, although it is clear that MCI-C cases in studies with follow-up $\leq 24$ months converted to $\mathrm{AD}$ in less than 24 months, it is possible that some MCI-C cases in studies with follow-up $>24$ months also converted to AD before the threshold of 24 months. Finally, sensitivity and specificity values above $80 \%$ were considered indicative of optimal predictive performance according to international recommendations (The Ronald and Nancy Reagan Research Institute of the Alzheimer's Association and the national Institute on Aging working Group, 1998). Higher levels are not easy to be achieved given that analyses are derived from clinically diagnosed $\mathrm{AD}$ cases in which the diagnostic accuracy already approximates $85 \%$ when validated by the standard pathologic diagnosis at autopsy (Mendez et al., 1992; Victoroff et al., 1995). None of the studies included in this meta-analysis performed postmortem $\mathrm{AD}$ confirmation. It is thus necessary to test CSF biomarkers' predictive performance in pathologically confirmed $\mathrm{AD}$ patients.

In conclusion, this study contributes to define several situations in which the CSF biomarkers seem to be clinically useful for predicting conversion from MCI to AD. In particular, a baseline CSF test result indicating $A \beta_{42} / p$-tau pathological values in MCI patients younger than 70 years has a moderate increase in the likelihood of developing AD. Moreover, a baseline CSF test result indicating pathological levels of p-tau increases the likelihood of developing $\mathrm{AD}$ within the next 24 months. To move forward in 
the knowledge about how different confounding factors influence the diagnostic and predictive performance of the CSF biomarkers is of utmost importance. Such knowledge will help the elaboration of a map of situations where the CSF biomarkers are useful, so that clinicians and researchers know when the new diagnostic criteria for MCI will be successful or otherwise prone to mistakes. This will be crucial when new disease-modifying treatments are available in the near future. Early prediction of MCI conversion to $\mathrm{AD}$ is expected to maximize treatment benefit if applied to the right people and before neuronal degeneration is too widespread and patients are already demented. In addition, this has ethical benefits because it is preferred not to treat patients with low risk of $\mathrm{AD}$ in trials that could cause side effects. Finally, this will also be important to enrich the samples with pure $\mathrm{AD}$ cases, both for research and clinical trials.

\section{ACKNOWLEDGMENTS}

The authors would like to thank the Spanish Ministry of Health, Social Services, and Equality; the Swedish Brain Power; the Strategic Research Programme in Neuroscience at Karolinska Institutet (StratNeuro); the Swedish Medical Society; and the regional agreement on medical training, clinical research (ALF) between Stockholm County Council and Karolinska Institutet.

\section{SUPPLEMENTARY MATERIAL}

The Supplementary Material for this article can be found online at http://www.frontiersin.org/Journal/10.3389/fnagi.2014.00287/ abstract

\section{REFERENCES}

Albert, M. S., DeKosky, S. T., Dickson, D., Dubois, B., Feldman, H. H., Fox, N. C., et al. (2011). The diagnosis of mild cognitive impairment due to Alzheimer's disease: recommendations from the National Institute on Aging-Alzheimer's Association workgroups on diagnostic guidelines for Alzheimer's disease. Alzheimers Dement. 7, 270-279. doi:10.1016/j.jalz.2011.03.008

Anoop, A., Singh, P. K., Jacob, R. S., and Maji, S. K. (2010). CSF biomarkers for Alzheimer's disease diagnosis. Int. J. Alzheimers Dis. 2010, 606802. doi:10.4061/2010/606802

Artero, S., Petersen, R. C., Touchon, J., and Ritchie, K. (2006). Revised criteria for mild cognitive impairment: validation within a longitudinal population study. Dement. Geriatr. Cogn. Disord. 22, 465-470. doi:10.1159/000096287

Bennett, D. A., Schneider, J. A., Arvanitakis, Z., Kelly, J. F., Aggarwal, N. T., Shah, R. C., et al. (2006). Neuropathology of older persons without cognitive impairment from two community-based studies. Neurology 66, 1837-1844. doi:10.1212/01.wnl.0000219668.47116.e6

Bloudek, L. M., Spackman, D. E., Blankenburg, M., and Sullivan, S. D. (2011). Review and meta-analysis of biomarkers and diagnostic imaging in Alzheimer's disease. JAD 26, 627-645. doi:10.3233/JAD-2011-110458

Bouwman, F. H., Schoonenboom, N. S., Verwey, N. A., van Elk, E. J., Kok, A., Blankenstein, M. A., et al. (2009). CSF biomarker levels in early and late onset Alzheimer's disease. Neurobiol. Aging 30, 1895-1901. doi:10.1016/j.neurobiolaging.2008.02. 007

Bouwman, F. H., Schoonenboom, S. N., van der Flier, W. M., van Elk, E. J., Kok, A., Barkhof, F., et al. (2007). CSF biomarkers and medial temporal lobe atrophy predict dementia in mild cognitive impairment. Neurobiol. Aging 28, 1070-1074. doi:10.1016/j.neurobiolaging.2006.05.006

Brys, M., Pirraglia, E., Rich, K., Rolstad, S., Mosconi, L., Switalski, R., et al. (2009). Prediction and longitudinal study of CSF biomarkers in mild cognitive impairment. Neurobiol. Aging 30, 682-690. doi:10.1016/j.neurobiolaging.2007.08.010

Buchhave, P., Minthon, L., Zetterberg, H., Wallin, A. K., Blennow, K., and Hansson, O. (2012). Cerebrospinal fluid levels of $\beta$-amyloid 1-42, but not of tau, are fully changed already 5 to 10 years before the onset of Alzheimer dementia. Arch. Gen Psychiatry 69, 98-106. doi:10.1001/archgenpsychiatry.2011.155

Choo, I. H., Ni, R., Schöll, M., Wall, A., Almkvist, O., and Nordberg, A. (2013). Combination of (18)F-FDG PET and cerebrospinal fluid biomarkers as a better predictor of the progression to Alzheimer's disease in mild cognitive impairment patients. JAD 33, 929-939. doi:10.3233/JAD-2012-121489

Deeks, J., Altman, D., and Bradburn, M. (2001). "Statistical methods for examining heterogeneity and combining results from several studies in meta-analysis," in Systematic Reviews in Health Care: Meta-analysis in Context, 2nd Edn, eds M. Egger, G. Davey Smith, and D. G. Altman (London: BMJ Publ. Gr), 285-312.

DerSimonian, R., and Laird, N. (1986). Meta-analysis in clinical trials. Control. Clin. Trials 7, 177-188. doi:10.1016/0197-2456(86)90046-2

Diniz, B. S. O., Pinto Júnior, J. A., and Forlenza, O. V. (2008). Do CSF total tau, phosphorylated tau, and beta-amyloid 42 help to predict progression of mild cognitive impairment to Alzheimer's disease? A systematic review and meta-analysis of the literature. World J. Biol. Psychiatry 9, 172-182. doi:10.1080/15622970701535502

Eckerström, C., Andreasson, U., Olsson, E., Rolstad, S., Blennow, K., Zetterberg, H., et al. (2010). Combination of hippocampal volume and cerebrospinal fluid biomarkers improves predictive value in mild cognitive impairment. Dement. Geriatr. Cogn. Disord. 29, 294-300. doi:10.1159/000289814

Ewers, M., Buerger, K., Teipel, S. J., Scheltens, P., Schröder, J., Zinkowski, R. P., et al. (2007). Multicenter assessment of CSF-phosphorylated tau for the prediction of conversion of MCI. Neurology 69, 2205-2212. doi:10.1212/01.wnl.0000286944. 22262.ff

Ewers, M., Walsh, C., Trojanowski, J. Q., Shaw, L. M., Petersen, R. C., Jack, C. R. Jr, et al. (2012). Prediction of conversion from mild cognitive impairment to Alzheimer's disease dementia based upon biomarkers and neuropsychological test performance. Neurobiol. Aging 33, 1203-1214. doi:10.1016/j.neurobiolaging. 2010.10.019

Fagan, A. M., Shaw, L. M., Xiong, C., Vanderstichele, H., Mintun, M. A., Trojanowski, J. Q., et al. (2011). Comparison of analytical platforms for cerebrospinal fluid measures of $\beta$-amyloid 1-42, total tau, and p-tau 181 for identifying Alzheimer disease amyloid plaque pathology. Arch. Neurol. 68, 1137-1144. doi:10.1001/archneurol.2011.105

Ferreira, D., Perestelo-Pérez, L., Westman, E., Wahlund, L.-O., and Sarría, A., Serrano-Aguilar, P. (2014). Meta-review of CSF core biomarkers in Alzheimer's disease: the state-of-the-art after the new revised diagnostic criteria. Front. Aging Neurosci. 6:1-24. doi:10.3389/fnagi.2014.00047

Galluzzi, S., Geroldi, C., Amicucci, G., Bocchio-Chiavetto, L., Bonetti, M., Bonvicini, C., et al. (2013). Supporting evidence for using biomarkers in the diagnosis of MCI due to AD. J. Neurol. 260, 640-650. doi:10.1007/s00415-012-6694-0

Gaser, C., Franke, K., Klöppel, S., Koutsouleris, N., and Sauer, H. (2013). Brain AGE in mild cognitive impaired patients: predicting the conversion to Alzheimer's disease. PLoS ONE 8:e67346. doi:10.1371/journal.pone.0067346

Green, M. S., Kaye, J. A., and Ball, M. J. (2000). The Oregon Brain Aging Study: Neuropathology accompanying healthy aging in the oldest old. Neurology 54, 105-113. doi:10.1212/WNL.54.1.105

Hampel, H., Teipel, S. J., Fuchsberger, T., Andreasen, N., Wiltfang, J., Otto, M., et al. (2004). Value of CSF B-amyloid $1-42$ and tau as predictors of Alzheimer's disease in patients with mild cognitive impairment. Mol. Psychiatry 9, 705-710. doi:10.1038/sj.mp.4001473

Hansson, O., Zetterberg, H., Buchhave, P., Andreasson, U., Londos, E., Minthon, L., et al. (2007). Prediction of Alzheimer's disease using the CSF Abeta42/Abeta40 ratio in patients with mild cognitive impairment. Dement. Geriatr. Cogn. Disord. 23, 316-320. doi:10.1159/000100926

Hansson, O., Zetterberg, H., Buchhave, P., Londos, E., Blennow, K., and Minthon, L. (2006). Association between CSF biomarkers and incipient Alzheimer's disease in patients with mild cognitive impairment: a follow-up study. Lancet Neurol. 5, 228-234. doi:10.1016/S1474-4422(06)70355-6

Hardy, R., and Thompson, S. (1998). Detecting and describing heterogeneity in meta-analysis. Stat. Med. 17, 841-856. doi:10.1002/(SICI)1097-0258(19980430) 17:8<841::AID-SIM781>3.0.CO;2-D

Hertze, J., Minthon, L., Zetterberg, H., Vanmechelen, E., Blennow, K., and Hansson, O. (2010). Evaluation of CSF biomarkers as predictors of Alzheimer's disease: a clinical follow-up study of 4.7 years. JAD 21, 1119-1128. doi:10.3233/JAD-2010100207

Herukka, S.-K., Hallikainen, M., Soininen, H., and Pirttilä, T. C. S. F. (2005). AB42 and tau or phosphorylated tau and prediction of progressive 
mild cognitive impairment. Neurology 64, 1294-1297. doi:10.1212/01.WNL. 0000156914.16988 .56

Holtzman, D. M. (2011). CSF biomarkers for Alzheimer's disease: current utility and potential future use. Neurobiol. Aging 32, S4-S9. doi:10.1016/j.neurobiolaging. 2011.09 .003

Hulstaert, F., Blennow, K., Ivanoiu, A., Schoonderwaldt, H.C., Riemen-Schneider, M., De Deyn, P. P., et al. (1999). Improved discrimination of AD patients using beta-amyloid(1-42) and Tau levels in CSF. Neurology 52, 1555-1562. doi:10.1212/WNL.52.8.1555

Jong, D., Jansen, W. M. M., Kremer, B. P. H., and Verbeek, M. M. (2006). Cerebrospinal fluid amyloid beta42/phosphorylated Tau ratio discriminates between Alzheimer's disease and vascular dementia. J. Gerontol. 61, 755-758. doi:10.1093/gerona/61.7.755

Liberati, A., Altman, D. G., Tetzlaff, J., Mulrow, C., Gøtzsche, P. C., Ioannidis, J. P., et al. (2009). The PRISMA statement for reporting systematic reviews and meta-analyses of studies that evaluate health care interventions: explanation and elaboration. Ann. Intern. Med. 151, 65-94. doi:10.7326/0003-4819-151-4200908180-00136

Maddalena, A., Papassotiropoulos, A., Müller-Tillmanns, B., Jung, H. H., Hegi, T., Nitsch, R. M., et al. (2003). Biochemical diagnosis of Alzheimer disease by measuring the cerebrospinal fluid ratio of phosphorylated tau protein to beta-amyloid peptide 42. Arch. Neurol. 60, 1202-1206. doi:10.1001/archneur.60. 9.1202

Mattsson, N., Andreasson, U., Persson, S., Arai, H., Batish, S. D., Bernardini, S., et al. (2011). The Alzheimer's association external quality control program for cerebrospinal fluid biomarkers. Alzheimers Dement. 7, 386-395. doi:10.1016/j.jalz. 2011.05.2243

Mattsson, N., Rosén, E., Hansson, O., Andreasen, N., Parnetti, L., Jonsson, M., et al. (2012). Age and diagnostic performance of Alzheimer disease CSF biomarkers. Neurology 78, 468-476. doi:10.1212/WNL.0b013e3182477eed

Mattsson, N., Zetterberg, H., Hansson, O., Andreasen, N., Parnetti, L., Jonsson, M., et al. (2009). CSF biomarkers and incipient Alzheimer disease in patients with mild cognitive impairment. JAMA 302, 385-393. doi:10.1001/jama.2009. 1064

Mendez, M., Mastri, A., Sung, J., and Frey, W. (1992). Clinically diagnosed Alzheimer disease: neuropathologic findings in 650 cases. Alzheimer Dis. Assoc. Disord. 6, 35-43. doi:10.1097/00002093-199205000-00004

Mitchell, A. J. C. S. F. (2009). Phosphorylated tau in the diagnosis and prognosis of mild cognitive impairment and Alzheimer's disease - a meta-analysis of 51 studies. J Neurol. Neurosurg. Psychiatr. 80, 966-975. doi:10.1136/jnnp.2008. 167791

Moher, D., Liberati, A., Tetzlaff, J., Altman, D., and the PRISMA Group. (2010). Preferred reporting items for systematic reviews and meta-analyses: the PRISMA statement. Int. J. Surg. 8, 336-341. doi:10.1016/j.ijsu.2010.02.007

Monge-Argilés, J. A., Muñoz-Ruiz, C., Pampliega-Pérez, A., Gómez-López, M. J., Sánchez-Payá, J., Rodríguez Borja, E., et al. (2011). Biomarkers of Alzheimer's disease in the cerebrospinal fluid of Spanish patients with mild cognitive impairment. Neurochem. Res. 36, 986-993. doi:10.1007/s11064-011-0438-x

Monge-Argilés, J. A., Sánchez-Payá, J., Muñoz-Ruiz, C., Pampliega-Pérez, A., Montoya-Gutiérrez, J., and Leiva-Santana, C. (2010). Biomarkers in the cerebrospinal fluid of patients with mild cognitive impairment: a meta-analysis of their predictive capacity for the diagnosis of Alzheimer's disease. Rev. Neurol. 50, 193-200.

Parnetti, L., Chiasserini, D., Eusebi, P., Giannandrea, D., Bellomo, G., De Carlo, C., et al. (2012). Performance of a $\beta 1-40$, a $\beta 1-42$, total tau, and phosphorylated tau as predictors of dementia in a cohort of patients with mild cognitive impairment. JAD 29, 229-238. doi:10.3233/JAD-2011-111349

Parnetti, L., Lanari, A., Silvestrelli, G., Saggese, E., and Reboldi, P. (2006). Diagnosing prodromal Alzheimer's disease: role of CSF biochemical markers. Mech. Ageing Dev. 127, 129-132. doi:10.1016/j.mad.2005.09.022

Petersen, R. C. (2004). Mild cognitive impairment as a diagnostic entity. J. Intern. Med. 256, 183-194. doi:10.1111/j.1365-2796.2004.01388.x

Petersen, R. C., Smith, G. E., Waring, S. C., Ivnik, R. J., Tangalos, E. G., and Kokmen, E. (1999). Mild cognitive impairment: clinical characterization and outcome. Arch. Neurol. 56, 303-308. doi:10.1001/archneur.56.3.303

Petersen, R. C., Doody, R., Kurz, A., Mohs, R. C., Morris, J. C., Rabins, P. V., et al. (2001). Current concepts in mild cognitive impairment. Arch. Neurol. 58, 19851992. doi:10.1001/archneur.58.12.1985
Prestia, A., Caroli, A., van der Flier, W. M., Ossenkoppele, R., Van Berckel, B., Barkhof, F., et al. (2013). Prediction of dementia in MCI patients based on core diagnostic markers for Alzheimer disease. Neurology 80, 1048-1056. doi:10.1212/WNL.0b013e3182872830

Qizilbash, N. (ed.) (2002). Evidenced-based Dementia Practice. Oxford: Blackwell Publishing.

Ritchie, K., Artero, S., and Touchon, J. (2001). Classification criteria for mild cognitive impairment: a population-based validation study. Neurology 56, 37-42. doi:10.1212/WNL.56.1.37

Roe, C. M., Fagan, A. M., Grant, E. A., Hassenstab, J., Moulder, K. L., Maue Dreyfus, D., et al. (2013). Amyloid imaging and CSF biomarkers in predicting cognitive impairment up to 7.5 years later. Neurology 80, 1784-1791. doi:10.1212/WNL.0b013e3182918ca6

Shaffer, J. L., Petrella, J. R., Sheldon, F. C., Choudhury, K. R., Calhoun, V. D., Coleman, R. E., et al. (2013). Predicting cognitive decline in subjects at risk for Alzheimer disease by using combined cerebrospinal fluid, MR imaging, and PET biomarkers. Radiology 266, 583-591. doi:10.1148/radiol.12120010

Shaw, L. M., Vanderstichele, H., Knapik-Czajka, M., Clark, C. M., Aisen, P. S., Petersen, R. C., et al. (2009). Cerebrospinal fluid biomarker signature in Alzheimer's disease neuroimaging initiative subjects. Ann. Neurol. 65, 403-413. doi:10.1002/ana.21610

Sjögren, M., Vanderstichele, H., Agren, H., Zachrisson, O., Edsbagge, M., Wikkelso, C., et al. (2001). Tau and Ab42 in cerebrospinal fluid from healthy adults 21-93 years of age: establishment of reference values. Clin. Chem. 47, 1776-1781.

The Ronald and Nancy Reagan Research Institute of the Alzheimer's Association and the national Institute on Aging working Group. (1998). Consensus report of the working group on: "molecular and biochemical markers of Alzheimer's disease. Neurobiol. Aging 19, 109-116.

Toledo, J. B., Korff, A., Shaw, L. M., Trojanowski, J. Q., and Zhang, J. C. S. F. (2013). $\alpha$-Synuclein improves diagnostic and prognostic performance of CSF tau and A $\beta$ in Alzheimer's disease. Acta Neuropathol. 126, 683-697. doi:10.1007/s00401013-1148-z

van Harten, A. C., Kester, M. I., Visser, P. J., Blankenstein, M. A., Pijnenburg, Y. A., van der Flier, W. M., et al. (2011). Tau and p-tau as CSF biomarkers in dementia: a meta-analysis. Clin. Chem. Lab. Med. 49, 353-366. doi:10.1515/CCLM.2011.086

Victoroff, J., Mack, W., Lyness, S., and Chui, H. (1995). Multicenter Clinicopathological Correlation in Dementia. Am. J. Psychiatry 152, 1476-1484.

Vos, S., van Rossum, I., Burns, L., Knol, D., Scheltens, P., Soininen, H., et al. (2012). Test sequence of CSF and MRI biomarkers for prediction of AD in subjects with MCI. Neurobiol. Aging 33, 2272-2281. doi:10.1016/j.neurobiolaging.2011. 12.017

Vos, S. J., van Rossum, I. A., Verhey, F., Knol, D. L., Soininen, H., Wahlund, L. O., et al. (2013). Prediction of Alzheimer disease in subjects with amnestic and nonamnestic MCI. Neurology 80, 1124-1132. doi:10.1212/WNL.0b013e318288690c

Westman, E., Muehlboeck, J.-S., and Simmons, A. (2012). Combining MRI and CSF measures for classification of Alzheimer's disease and prediction of mild cognitive impairment conversion. Neuroimage 62, 229-238. doi:10.1016/j. neuroimage.2012.04.056

Whiting, P., Rutjes, A., Westwood, M., Mallett, S., Deeks, J. J., Reitsma, J. B., et al. (2011). QUADAS-2: a revised tool for the quality assessment of diagnostic accuracy studies. Ann. Intern. Med. 155, 529-536. doi:10.7326/0003-4819-155-8201110180-00009

Winblad, B., Palmer, K., Kivipelto, M., Jelic, V., Fratiglioni, L., Wahlund, L. O., et al. (2004). Mild cognitive impairment-beyond controversies, towards a consensus: report of the International Working Group on Mild Cognitive Impairment. J. Intern. Med. 256, 240-246. doi:10.1111/j.1365-2796.2004.01380.x

Zamora, J., Abraira, V., Muriel, A., Khan, K., and Coomarasamy, A. (2006). MetaDiSc: a software for meta-analysis of test accuracy data. BMCMed. Res. Methodol. 6:31. doi:10.1186/1471-2288-6-31

Zetterberg, H., Wahlund, L. O., and Blennow, K. (2003). Cerebrospinal fluid markers for prediction of Alzheimer's disease. Neurosci. Lett. 352, 67-69. doi:10.1016/j.neulet.2003.08.011

Conflict of Interest Statement: The authors declare that the research was conducted in the absence of any commercial or financial relationships that could be construed as a potential conflict of interest. 
Received: 24 July 2014; accepted: 29 September 2014; published online: 16 October 2014.

Citation: Ferreira D, Rivero-Santana A, Perestelo-Pérez L, Westman E, Wahlund L-O, Sarría A and Serrano-Aguilar P (2014) Improving CSF biomarkers' performance for predicting progression from Mild Cognitive Impairment to Alzheimer's disease by considering different confounding factors: a meta-analysis. Front. Aging Neurosci. 6:287. doi: $10.3389 /$ fnagi.2014.00287

This article was submitted to the journal Frontiers in Aging Neuroscience.
Copyright (๑) 2014 Ferreira, Rivero-Santana, Perestelo-Pérez, Westman, Wahlund, Sarría and Serrano-Aguilar. This is an open-access article distributed under the terms of the Creative Commons Attribution License (CC BY). The use, distribution or reproduction in other forums is permitted, provided the original author(s) or licensor are credited and that the original publication in this journal is cited, in accordance with accepted academic practice. No use, distribution or reproduction is permitted which does not comply with these terms. 